\title{
Influence of HOTAIR rs920778 and rs12826786 genetic variants on prostate cancer risk and progression-free survival
}

\author{
Ana Isabel Oliveira ${ }^{\ddagger 1,2}$, Ana Xavier-Magalhães ${ }^{\ddagger}, 1,2$, Catarina Moreira-Barbosa ${ }^{3}$, Hugo \\ Magalhães $^{1,2}$, Rui Henrique ${ }^{3,4,5}$, Carmen Jerónimo ${ }^{3,4}$ \& Bruno M Costa ${ }^{* 1,2}$ \\ ${ }^{1}$ Life \& Health Sciences Research Institute (ICVS), School of Medicine, Campus de Gualtar, University of Minho, $4710-057$ Braga, \\ Portugal \\ ${ }^{2}$ ICVS/3B's-PT Government Associate Laboratory, Braga/Guimarães, Campus de Gualtar, University of Minho, $4710-057$ Braga, \\ Portugal \\ ${ }^{3}$ Cancer Biology \& Epigenetics Group - Research Center CI-IPOP, Portuguese Oncology Institute of Porto, Porto, Portugal \\ ${ }^{4}$ Department of Pathology \& Molecular Immunology, Institute of Biomedical Sciences Abel Salazar - University of Porto \\ (ICBAS-UP), Porto, Portugal \\ ${ }^{5}$ Department of Pathology, Portuguese Oncology Institute of Porto (IPO Porto), Porto, Portugal \\ * Author for correspondence: Tel.: +351 2536048 72; Fax: +351 2536048 31; bfmcosta@med.uminho.pt \\ $\ddagger$ Authors contributed equally
}

\begin{abstract}
Aim: Evaluate the impact of the single nucleotide polymorphisms rs920778 and rs12826786 in the long noncoding RNA HOTAIR in the susceptibility and prognosis of prostate cancer (PCa) patients. Patients \& methods: HOTAIR single nucleotide polymorphisms were genotyped by restriction fragment length polymorphism in 151 PCa cases and 180 cancer-free controls. Odds ratio, 95\% Cls and prognostic significance were calculated. Results: Our data showed no statistically significant associations between HOTAIR polymorphic variants in rs920778 and rs12826786 and PCa susceptibility. However, the CC genotype in rs 12826786 was significantly associated with shorter biochemical recurrence-free survival in pT3-stage PCa patients. Conclusion: Our results indicate that HOTAIR rs12826786 CC genotype may be an independent prognostic biomarker in a particular subset of PCa tumors.
\end{abstract}

First draft submitted: 9 August 2017; Accepted for publication: 21 November 2017; Published online: 13 February 2018

Keywords: HOTAIR • PCa • rs12826786•rs920778 • SNPS

Prostate cancer ( $\mathrm{PCa})$ is the second most common malignancy diagnosed in men worldwide, and one of the major causes of cancer-related morbidity and mortality [1]. Its classification is based on the Gleason grading system [2,3], which takes into account the glandular architecture of the tumor and the tumor node metastasis staging system [4]. Ranging from clinically indolent to extremely aggressive tumors, PCa is known for its remarkable heterogeneity, and is a challenge for clinicians to predict when a tumor will recur or progress, and which invasive cancers will eventually metastasize and be fatal [5]. Few factors have been associated with increased PCa risk, and there are only three well-established risk factors for the development of the disease: family history, ethnicity and age [6]. Additionally, genome-wide and replication association studies have shown that particular genetic variants in single nucleotide polymorphisms (SNPs) may also be useful predictors of PCa risk [7].

Since the discovery that the human transcriptome is more complex than a collection of protein-coding genes and their splice variants [8], it became crucial to understand the functions of nonprotein coding regions [9]. Among these regions, long noncoding RNAs (lncRNA) represent spliced, polyadenylated and about 200 nucleotide-long RNAs, which are crucial in physiological and pathologic conditions, including in cancer [10]. HOTAIR is a transacting lncRNA whose aberrant expression has been associated with cancer patients' overall survival, metastatic potential, tumor recurrence and chemotherapy response in many tumor types [11-13]. Recently, HOTAIR has been suggested to play an oncogenic role in PCa [14]. Additionally, its ability to bind and reduce androgen receptor degradation and its enhanced transcriptional activity were shown to potentiate castration-resistant prostate cancer cell growth [15]. The relevance of particular HOTAIR genetic polymorphisms in cancer risk has been established in a variety of 
Table 1. Clinicopathologic features of prostate cancer patients and cancer-free controls.

\begin{tabular}{|lll|}
\hline Clinicopathologic feature & Cases & \multicolumn{1}{l}{ Controls } \\
\hline Number of cases & 151 & 180 \\
\hline Age (years), mean \pm SD & $64.63 \pm 5.33$ & $53.07 \pm 16.93$ \\
\hline PSA $(\mathrm{ng} / \mathrm{ml})$, mean \pm SD & $8.9 \pm 3.6$ & Not applicable \\
\hline PSA grade, $\mathrm{n}(\%)$ & & Not applicable \\
\hline$<8$ & $71(47)$ & Not applicable \\
\hline$\geq 8$ to $<15$ & $66(44)$ & Not applicable \\
\hline$\geq 15$ & $14(9)$ & Not applicable \\
\hline Gleason score, $n(\%)$ & & Not applicable \\
\hline$<7$ (grade group 1$)^{\dagger}$ & $36(24)$ & Not applicable \\
\hline$=7$ (grade groups 2 and 3$)^{\dagger}$ & $74(49)$ & Not applicable \\
\hline$>7$ (grade groups 4 and 5$)^{\dagger}$ & $41(27)$ & Not applicable \\
\hline Pathological stage, $n(\%)$ & & Not applicable \\
\hline pT2 & $65(43)$ & Not applicable \\
\hline pT3 & $86(57)$ & Not applicable \\
\hline$\dagger$ Grade groups, based on 2016 WHO classification of prostate tumors. & \\
\hline SD: Standard deviation. & & \\
\hline
\end{tabular}

tumor types, including colorectal [16], breast [17], esophageal [18], gastric [19] and brain [20] cancers. In particular, variants in SNPs, rs920778 (located in an intronic enhancer) and rs12826786 (within the HOTAIR promoter), functionally affect HOTAIR gene expression levels [18,19,21].

In the context of the emerging evidences for roles of HOTAIR in prostate carcinogenesis [14,15], we evaluated how these two functional HOTAIR SNPs may associate with PCa risk and patient prognosis in a Caucasian population.

\section{Patients \& methods}

\section{Study population}

This case-control study comprised samples obtained from 151 PCa patients diagnosed at Portuguese Oncology Institute - Porto, between 1999 and 2008. For controls, 180 cancer-free male individuals selected from blood donors previously characterized for HOTAIR rs920778 and rs12826786 polymorphisms (130 cases) [20] and from the Portuguese Oncology Institute (50 cases; healthy PCa-free individuals, confirmed with negative biopsy results) were included. All participants were of Caucasian ethnic background. All patients enrolled in this study were clinically stage I or II at diagnosis and were submitted to radical prostatectomy. Tumors were classified by routine histopathological examination by an expert pathologist, and scored for Gleason [2,22] and pathological staging [23]. The study was conducted according to institutional ethical standards, and all subjects provided signed informed consent to participate in research studies (CES-IPOPFG-EPE 019/08). The clinicopathological data are summarized in Table 1.

\section{SNP genotyping}

Genomic DNA was collected from peripheral blood leukocytes by proteinase K/phenol-chloroform/ethanol treatment. In brief, samples were digested overnight at $55^{\circ} \mathrm{C}$ in $300 \mu \mathrm{l}$ of $10 \%$ sodium dodecyl sulfate and proteinase $\mathrm{K}$ $(20 \mathrm{mg} / \mathrm{ml}, 25 \mu \mathrm{l})$. The DNA from clinical samples was extracted by phenol-chloroform and ethanol precipitated. DNA concentration and purity were determined using NanoDrop Lite Spectrophotometer (Nanodrop Technologies, Thermo Fisher, DE, USA). HOTAIR SNPs rs920778 (C>T) and rs12826786 (C>T) were genotyped through PCR-based restriction fragment length polymorphism. Fifty nanograms of DNA were used for PCR amplification with KAPA Taq DNA Polymerase (Kapa Biosystems, MA, USA). Primers sequences and PCR conditions were previously described by us [20] and based on earlier publications [19,24]. PCR products were enzymatically digested at $37^{\circ} \mathrm{C}$ for 5 min with FastDigest MspI (Thermo Scientific, MA, USA) for rs920778, or 30 min with FastDigest BglII (Thermo Scientific) for rs12826786. Digestion products were resolved in 4\% agarose gel stained with GreenSafe Premium (NZYtech, Lisboa, Portugal). 


\begin{tabular}{|c|c|c|c|c|c|c|c|}
\hline Polymorphism & Control & $\mathrm{PCa}$ & OR $(95 \% \mathrm{Cl})^{\dagger}$ & $\begin{array}{l}\text { Gleason score }>7 \\
(\text { grade groups } 4 \text { and } 5)^{\ddagger}\end{array}$ & OR $(95 \% \mathrm{Cl})^{\dagger}$ & $\begin{array}{l}\text { Pathological stage } \\
\text { pT3 }\end{array}$ & OR $(95 \% \mathrm{Cl})^{\dagger}$ \\
\hline \multicolumn{8}{|l|}{ rs920778 } \\
\hline \multicolumn{8}{|l|}{ Genotypes } \\
\hline TT & 81 & 78 & - & 21 & - & 43 & - \\
\hline CT & 72 & 50 & $0.721(0.448-1.161)$ & 14 & $0.750(0.355-1.583)$ & 27 & $0.706(0.397-1.257)$ \\
\hline CC & 27 & 23 & $0.885(0.468-1.673)$ & 6 & $0.857(0.313-2.345)$ & 16 & $1.116(0.543-2.294)$ \\
\hline $\mathrm{CC}+\mathrm{CT}$ & 99 & 73 & $0.766(0.496-1.182)$ & 20 & $0.779(0.395-1.537)$ & 43 & $0.818(0.489-1.369)$ \\
\hline \multicolumn{8}{|l|}{ Alleles } \\
\hline $\mathrm{T}$ & 234 & 206 & - & 56 & - & 113 & - \\
\hline c & 126 & 96 & $0.865(0.625-1.198)$ & 26 & $0.862(0.516-1.440)$ & 59 & $0.970(0.662-1.421)$ \\
\hline \multicolumn{8}{|l|}{ rs12826786 } \\
\hline \multicolumn{8}{|l|}{ Genotypes } \\
\hline CC & 88 & 85 & - & 22 & - & 48 & - \\
\hline CT & 71 & 51 & $0.744(0.466-1.187)$ & 15 & $0.845(0.408-1.748)$ & 28 & $0.723(0.412-1.267)$ \\
\hline TT & 21 & 15 & $0.739(0.358-1.529)$ & 4 & $0.762(0.237-2.447)$ & 10 & $0.873(0.380-2.004)$ \\
\hline $\mathrm{TT}+\mathrm{CT}$ & 92 & 66 & $0.743(0.481-1.147)$ & 19 & $0.826(0.419-1.630)$ & 38 & $0.757(0.452-1.269)$ \\
\hline \multicolumn{8}{|l|}{ Alleles } \\
\hline c & 247 & 221 & - & 59 & - & 124 & - \\
\hline T & 113 & 81 & $0.801(0.571-1.124)$ & 23 & $0.852(0.501-1.449)$ & 48 & $0.846(0.567-1.263)$ \\
\hline
\end{tabular}

\section{Statistical analyses}

Statistical analyses were performed using SPSS 22.0 (IBM SPSS Statistics, IBM ${ }^{\circledR}$, IL, USA). Allele and genotype frequencies in PCa patients and cancer-free controls were compared by $\chi^{2}$ test, and nonparametric WilcoxonMann-Whitney test was used to compare age distribution between groups. Hardy-Weinberg equilibrium was evaluated by $\chi^{2}$, comparing the expected and observed genotype frequencies of HOTAIR SNPs in the control group. Odds ratio and $95 \%$ CIs were calculated by univariable and multivariable logistic regression (adjusted for age as a continuous variable). The influence of HOTAIR genetic variants in PCa patients' biochemical recurrencefree survival (RFS) (defined as prostate-specific antigen [PSA] values $>0.2 \mathrm{ng} / \mathrm{ml}$ following radical prostatectomy) was illustrated with Kaplan-Meier survival curves, and the differences assessed by log-rank test (univariable) and Cox regression (multivariable) model adjusted for patients' age as a continuous variable, initial PSA and Gleason grading (categorized in all cases using the recommendations of Epstein et al. [22], which has been then incorporated in the 2016 WHO classification guidelines). Statistical significance was considered for $\mathrm{p}$-values $<0.05$.

\section{Results}

\section{HOTAIR \& PCa risk}

We analyzed $151 \mathrm{PCa}$ patients and 180 cancer-free control males to study associations between variants in two HOTAIR polymorphisms (rs920778 and rs12826786) and PCa susceptibility. The distribution of rs920778 and rs12826786 allele frequencies in the control group was in Hardy-Weinberg equilibrium $(\mathrm{p}=0.272$ and $\mathrm{p}=0.512$, respectively). A summary of the clinicopathologic features of controls and cases is shown in Table 1 . The genotype and allele frequencies of the rs920778 and rs12826786 polymorphisms in controls and PCa cases are shown in Table 2. The frequencies of TT, CT and CC genotypes of rs920778 were 45.0, 40.0 and 15.0\% in cancer-free controls, and 51.7, 33.1 and 15.2\% in PCa patients, respectively, indicating no statistically significant differences ( $\mathrm{p}>0.05)$. In both groups, the $\mathrm{T}$ allele was the most frequent $(65.0 \%$ in the control group and $68.2 \%$ in the PCa samples). Regarding rs12826786 polymorphism, the frequencies of CC, CT and TT genotypes were 48.9, 39.4 and $11.7 \%$ in controls, and 56.3, 33.8 and 9.9\% in PCa cases, respectively, again indicating no statistically significant differences $(\mathrm{p}>0.05)$. The $\mathrm{C}$ allele was the most frequent $(68.6 \%$ in the control group and $73.2 \%$ in PCa patients). 

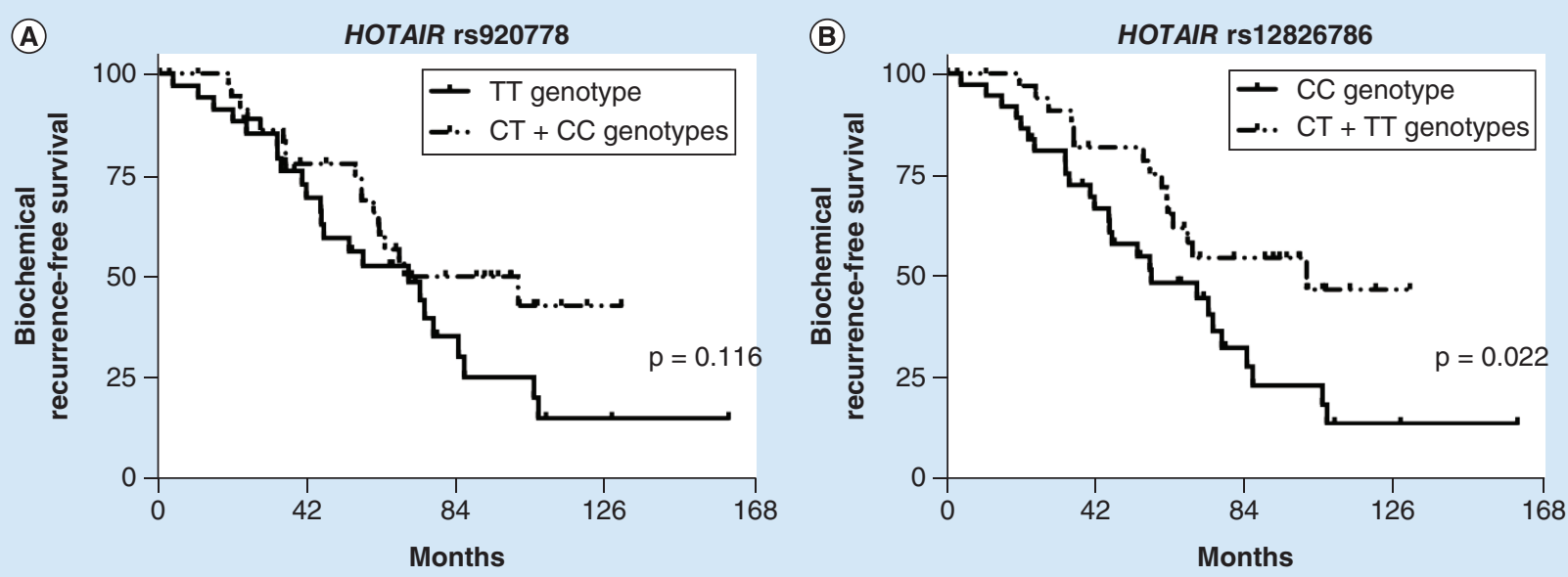

Figure 1. Effect of HOTAIR rs920778 and rs12826786 genetic variants in the biochemical recurrence-free survival of pT3 prostate cancer patients $(n=75)$. Kaplan-Meier curves illustrating biochemical recurrence-free survival defined by rs920778 (A) and rs12826786 (B) genotypes. (A) No statistical differences were observed between patients harboring different genotypes of rs920778 (Log-rank test, p-value $=0.116)$. (B) Patients harboring rs12826786 CC genotype $(n=40)$ have statistically significant shorter overall survival when compared with patients with CT and TT genotype $(n=35$; Log-rank test, $p$-value $=0.022)$. Tick marks indicate censored data.

Associations between the genotypes of HOTAIR rs920778 and rs12826786 and PCa susceptibility were first estimated using univariable analyses. Regarding rs920778, the $\mathrm{C}$ allele was not significantly associated with risk for PCa (Table 2). Similarly, genotypic analyses using TT as a reference (the most frequent genotype of the control group) showed that none of the other genotypes (CC, CT or CC + CT) were significantly associated with risk for PCa (Table 2). Regarding rs12826786, the T allele was not significantly associated with risk for PCa (Table 2). Genotype analyses using CC genotype as a reference showed that TT, CT and combined TT + CT genotypes were not significantly associated with increased risk for $\mathrm{PCa}$ (Table 2).

Similar analyses were done in the subset of PCa patients with more aggressive disease (two groups: Gleason score $>7$ or pathological stage pT3) against the control group. In the same line as the whole PCa dataset, no associations were observed in this subgroup of patients between rs920778 and rs 12826786 alleles, or genotype variants, and PCa risk (Table 2). These results suggest that HOTAIR rs920778 and rs12826786 variants do not confer risk to develop PCa or specific subtypes of aggressive PCa (Gleason score $>7$ or pT3).

A multivariable logistic regression model adjusted for patient age as a continuous variable was also applied for both polymorphisms. Consistent with the results observed in the univariable analysis, no associations between each polymorphic variant and risk for developing PCa were found (data not shown). As expected, increased age was found to be associated with increased risk for developing PCa (odds ratio: 1.070; 95\% CI: 1.050-1.092).

\section{Survival \& prognostic value of HOTAIR SNPS}

To understand the relevance of these HOTAIR SNPs on the prognosis of PCa patients, we evaluated how rs 920778 and rs12826786 genotypes associated with the progression-free survival (as assessed by biochemical recurrence) in pT2 $(\mathrm{n}=62)$ and pT3 $(\mathrm{n}=75)$ stage PCa patients with available follow-up data. For pT2 patients, no statistically significant associations were found (data not shown). Also, Kaplan-Meier curves with univariable logrank test (Figure 1) or multivariable Cox regression models (Table 3) showed no statistically significant associations between rs920778 polymorphism and pT3 PCa patients' biochemical RFS. In contrast, pT3 patients carrying the CC genotype in rs12826786 $(\mathrm{n}=40)$ showed statistically significant shorter biochemical RFS compared with all other patients (CT + TT genotypes, $\mathrm{n}=35$ ), both in univariable (Figure 1B) (Log rank test, $\mathrm{p}=0.022$ ) and in multivariable analyses adjusted for patient age, initial PSA and Gleason grading (Table 3) (Cox regression model, $p=0.018$ ), suggesting the $C$ variant in rs 12826786 as a negative and independent prognostic biomarker. Strengthening our findings, the $\mathrm{T}$ allele seems to be associated with a good prognosis, as the TT genotype conferred longer overall biochemical RFS comparing to CC $(\mathrm{p}=0.040)$. 
Table 3. Multivariable Cox regression analysis of the association between rs920778 and rs12826786 polymorphisms and biochemical recurrence-free survival for prostate cancer patients (pathological stage pT3).

\begin{tabular}{|c|c|c|c|}
\hline Polymorphism & Pathological Stage pT3 & $\operatorname{HR}(95 \% \mathrm{Cl})^{\dagger}$ & p-value \\
\hline \multicolumn{4}{|c|}{ rs920778 genotypes } \\
\hline TT & 37 & - & - \\
\hline $\mathrm{CC}$ & 14 & $0.619(0.236-1.625)$ & 0.330 \\
\hline $\mathrm{CT}$ & 24 & $0.553(0.259-1.180)$ & 0.126 \\
\hline $\mathrm{CC}+\mathrm{CT}$ & 38 & $0.573(0.288-1.137)$ & 0.111 \\
\hline Age & & $1.007(0.945-1.073)$ & 0.823 \\
\hline \multicolumn{4}{|l|}{ PSA grade } \\
\hline \multicolumn{4}{|l|}{$<8$} \\
\hline 35 & - & - & \\
\hline$\geq 8$ to $<15$ & 30 & $0.938(0.457-1.924)$ & 0.861 \\
\hline$\geq 15$ & 10 & $1.149(0.448-2.952)$ & 0.772 \\
\hline \multicolumn{4}{|c|}{ ISUP/WHO grading system } \\
\hline 1 & 7 & - & - \\
\hline 2 & 24 & $1.047(0.283-3.874)$ & 0.945 \\
\hline 3 & 7 & $1.131(0.241-5.320)$ & 0.876 \\
\hline 4 & 16 & $1.015(0.242-4.258)$ & 0.984 \\
\hline 5 & 21 & $1.401(0.387-5.076)$ & 0.608 \\
\hline \multicolumn{4}{|c|}{ rs12826786 genotypes } \\
\hline CC & 40 & - & - \\
\hline $\mathrm{TT}$ & 9 & $0.209(0.047-0.933)$ & 0.040 \\
\hline CT & 26 & $0.490(0.236-1.016)$ & 0.055 \\
\hline $\mathrm{TT}+\mathrm{CT}$ & 35 & $0.422(0.207-0.860)$ & 0.018 \\
\hline Age & & $1.001(0.938-1.069)$ & 0.968 \\
\hline \multicolumn{4}{|l|}{ PSA grade } \\
\hline$<8$ & 35 & - & - \\
\hline$\geq 8$ to $<15$ & 30 & $0.945(0.457-1.953)$ & 0.879 \\
\hline$\geq 15$ & 10 & $1.225(0.471-3.184)$ & 0.677 \\
\hline \multicolumn{4}{|c|}{ ISUP/WHO grading system } \\
\hline 1 & 7 & - & - \\
\hline 2 & 24 & $0.942(0.253-3.508)$ & 0.929 \\
\hline 3 & 7 & $1.193(0.253-5.640)$ & 0.823 \\
\hline 4 & 16 & $0.886(0.209-3.758)$ & 0.869 \\
\hline 5 & 21 & $1.447(0.399-5.242)$ & 0.574 \\
\hline
\end{tabular}

\section{Discussion}

SNPs represent the most frequent genetic variants among individuals, potentially affecting gene expression, function, phenotypes and diseases [25]. The study of SNPs in cancer has been increasing concomitantly with the interest in cancer genetic susceptibility. Particularly, SNPs in genes known to be implicated in carcinogenic processes have been studied as they may affect susceptibility to several cancer types [26-28]. PCa is not an exception, and SNPs of several genes have been studied and identified as putative biomarkers of susceptibility, including genes involved in DNA repair pathways (XRCC4, PMSI, XRCC1 and XPD) [29,30] or growth pathways (VEGF, IGF1 and EGF) [30-32]. The role of the lncRNA HOTAIR in PCa has recently been addressed, which points it as a driver of tumor cell growth, invasion/metastasis, androgen-independent androgen receptor activity and castration-resistant prostate cancer progression [14,15,33]. The influence of three HOTAIR SNPs (rs12826786, rs1899663 and rs4759314) in PCa pathophysiology was recently reported in an Iranian population [34]. In the context of the functional relevance of rs920778 and rs12826786 SNPs regulating HOTAIR expression levels $[18,19]$, we provide here the first study 
evaluating putative associations between these HOTAIR SNPs and PCa susceptibility and prognosis in a Caucasian population.

Using univariable and multivariable statistical analyses, our data showed that rs920778 and rs12826786 are not significantly associated with PCa susceptibility (neither in all PCa patients nor in the subset of more aggressive Gleason score $>7$ or pathologic stage pT3 tumors). In accordance, it was previously showed that rs 920778 does not influence gastric cancer susceptibility [35]. In contrast, in PCa, rs12826786 T allele was associated with increased risks for benign prostate hyperplasia and PCa [34]. Additionally, other studies in esophageal squamous cell carcinoma, gastric cancer [18,21], gastric cardia adenocarcinoma or breast cancer $[17,19,36,37]$ suggested a role of these polymorphisms in cancer susceptibility $[17-19,21,36,37]$. These observations are likely to reflect tumor-specific carcinogenic processes that are not shared among these tumor types, and also due to ethnic differences among studies. In fact, a recent meta-analysis showed that HOTAIR SNP rs920778 was associated with gastric cancer and esophageal squamous cell carcinoma, but not with breast cancer, in Asians but not in Caucasians [38]. Nonetheless, limitations in the sample size of our and others' studies must not be ruled out, warranting further studies in larger cohorts to validate these findings.

Interestingly, regarding the relevance of these HOTAIR SNPs on patient prognosis, we found that within the subset of PCa patients with nonorgan-confined/locally advanced tumors (pT3), those carrying a CC genotype in rs12826786 have significantly shorter biochemical RFS than those presenting CT or TT genotypes, both in univariable and multivariable analyses. Of note, rs12826786 is located in the promoter region of HOTAIR suggesting its variants may influence HOTAIR's expression. Since expression of HOTAIR is a known biomarker of poor prognosis in $\mathrm{PCa}$ [14,15], it is of relevance to study how variants of this SNP may induce differential expression levels that can have biological roles in PCa. Furthermore, our results are of great clinical importance as they may aid clinicians to timely identify PCa patients submitted to radical prostatectomy who are at increased risk of local recurrence and disease progression, who might benefit from a closer follow-up or novel therapeutic strategies.

\section{Conclusion}

In conclusion, our findings suggest that HOTAIR rs920778 and rs 12826786 do not play a major role in susceptibility to $\mathrm{PCa}$, while rs 12826786 might have a prognostic value in locally advanced PCa (pT3 stage) patients. In the future, further independent studies are required to validate our findings in larger datasets, as well as in patients of different ethnic origins to further understand the relevance of HOTAIR rs920778 and rs12826786 polymorphisms in PCa pathophysiology.

\section{Summary points}

- The relevance of HOTAIR genetic variants in prostate cancer remains elusive.

- HOTAIR single nucleotide polymorphisms rs920778 and rs12826786 do not associate with prostate cancer risk.

- rs12826786 predicts shorter biochemical recurrence-free survival in pT3 patients.

\section{Acknowledgements}

The authors would like to acknowledge to all the patients and control subjects that kindly provided the biological samples for this study.

Financial \& competing interests disclosure

Fundação para a Ciência e Tecnologia (IF/00601/2012 to BM Costa; SFRH/BD/52287/2013 to Al Oliveira; SFRH/BD/88220/2012 to AX Magalhães), Fundação Calouste Gulbenkian (BM Costa), Liga Portuguesa Contra o Cancro (BM Costa) and Inter-University Doctoral Programme in Ageing and Chronic Disease (PhDOC; to Al Oliveira and AX Magalhães). Project co-financed by Programa Operacional Regional do Norte (ON.2-O Novo Norte), Quadro de Referência Estratégico Nacional (QREN), Fundo Europeu de Desenvolvimento Regional (FEDER) and a grant (74-CI-IPOP) from Research Center of Portuguese Oncology Institute of Porto (C Jerónimo). The authors have no other relevant affiliations or financial involvement with any organization or entity with a financial interest in or financial conflict with the subject matter or materials discussed in the manuscript apart from those disclosed.

No writing assistance was utilized in the production of this manuscript. 


\section{Ethical conduct of research}

The study was conducted according to institutional ethical standards, and with the 1964 Helsinki declaration and its later amendments or comparable ethical standards. All subjects provided signed informed consent to participate in research studies (CESIPOPFG-EPE 019/08).

\section{References}

Papers of special note have been highlighted as: $\bullet$ of interest; $\bullet \bullet$ of considerable interest

1. Ferlay J, Shin HR, Bray F, Forman D, Mathers C, Parkin DM. Estimates of worldwide burden of cancer in 2008: GLOBOCAN 2008. Int. J. Cancer 127(12), 2893-2917 (2010).

2. Epstein JI. Update on the Gleason grading system. Ann. Pathol. 31(Suppl. 5), S20-S26 (2011).

3. Gordetsky J, Epstein J. Grading of prostatic adenocarcinoma: current state and prognostic implications. Diagn. Pathol. 11, 25 (2016).

4. Edge SB, Compton CC. The American Joint Committee on Cancer: the 7 th edition of the AJCC cancer staging manual and the future of TNM. Ann. Surg. Oncol. 17(6), 1471-1474 (2010).

5. Roobol MJ, Carlsson SV. Risk stratification in prostate cancer screening. Nat. Rev. Urol. 10(1), 38-48 (2013).

6. Patel AR, Klein EA. Risk factors for prostate cancer. Nat. Clin. Pract. Urol. 6(2), 87-95 (2009).

7. Liu H, Wang B, Han C. Meta-analysis of genome-wide and replication association studies on prostate cancer. Prostate 71(2), 209-224 (2011).

8. Kapranov P, Willingham AT, Gingeras TR. Genome-wide transcription and the implications for genomic organization. Nat. Rev. Genet. $8(6), 413-423$ (2007).

9. Guttman M, Amit I, Garber M et al. Chromatin signature reveals over a thousand highly conserved large non-coding RNAs in mammals. Nature 458(7235), 223-227 (2009).

10. Gibb EA, Brown CJ, Lam WL. The functional role of long non-coding RNA in human carcinomas. Mol. Cancer 10(1), 38-55 (2011).

11. Hajjari M, Salavaty A. HOTAIR: an oncogenic long non-coding RNA in different cancers. Cancer Biol. Med. 12(1), 1-9 (2015).

12. Yu X, Li Z. Long non-coding RNA HOTAIR: a novel oncogene (review). Mol. Med. Rep. 12(4), 5611-5618 (2015).

13. Bhan A, Mandal SS. LncRNA HOTAIR: a master regulator of chromatin dynamics and cancer. Biochim. Biophys. Acta 1856(1), 151-164 (2015).

14. Chiyomaru T, Yamamura S, Fukuhara $S$ et al. Genistein inhibits prostate cancer cell growth by targeting miR-34a and oncogenic HOTAIR. PLoS ONE 8(8), e70372 (2013).

15. Zhang A, Zhao JC, Kim J et al. LncRNA HOTAIR enhances the androgen-receptor-mediated transcriptional program and drives castration-resistant prostate cancer. Cell Rep. 13(1), 209-221 (2015).

16. Xue Y, Gu D, Ma G et al. Genetic variants in lncRNA HOTAIR are associated with risk of colorectal cancer. Mutagenesis 30(2), 303-310 (2015).

17. Bayram S, Sümbül AT, Batmacı CY, Genç A. Effect of HOTAIR rs 920778 polymorphism on breast cancer susceptibility and clinicopathologic features in a Turkish population. Tumor Biol. 36(5), 3863-3870 (2015).

- Studies the relevance of HOTAIR single nucleotide polymorphisms in the development of cancer.

18. Zhang X, Zhou L, Fu G et al. The identification of an ESCC susceptibility SNP rs920778 that regulates the expression of lncRNA HOTAIR via a novel intronic enhancer. Carcinogenesis 35(9), 2062-2067 (2014).

\section{- Studies the impact of rs12826786 in the risk of gastric cardia adenocarcinoma.}

19. Guo W, Dong Z, Bai Y et al. Associations between polymorphisms of HOTAIR and risk of gastric cardia adenocarcinoma in a population of north China. Tumor Biol. 36(4), 2845-2854 (2015).

\section{- Addresses the impact of HOTAIR rs920778 and rs12826786 in the development of glioma.}

20. Xavier-Magalhaes A, Oliveira AI, De Castro JV et al. Effects of the functional HOTAIR rs920778 and rs 12826786 genetic variants in glioma susceptibility and patient prognosis. J. Neurooncol. 132(1), 27-34 (2017).

21. Pan W, Liu L, Wei J et al. A functional lncRNA HOTAIR genetic variant contributes to gastric cancer susceptibility. Mol. Carcinog. 55(1), 90-96 (2016).

22. Epstein JI, Amin MB, Reuter VE, Humphrey PA. Contemporary Gleason grading of prostatic carcinoma: an update with discussion on practical issues to implement the 2014 International Society of Urological Pathology (ISUP) consensus conference on Gleason grading of prostatic carcinoma. Am. J. Surg. Pathol. 41(4), e1-e7 (2017).

23. Sauter G, Epstein JI, Sesterhenn IA. Pathology and Genetics of Tumours of the Urinary System and Male Genital Organs. Eble JN (Ed.) IARC, Lyon, France 109 (2004).

24. Zhang X, Zhou L, Fu G et al. The identification of an ESCC susceptibility SNP rs 920778 that regulates the expression of lncRNA HOTAIR via a novel intronic enhancer. Carcinogenesis 35(9), 2062-2067 (2014).

25. Shastry BS. SNPs: impact on gene function and phenotype. Methods Mol. Biol. 578, 3-22 (2009). 
26. Salvi S, Conteduca V, Gurioli G, Calistri D, Casadio V, De Giorgi U. Impact of candidate genetic polymorphisms in prostate cancer: an overview. Mol. Diagn. Ther. 20(1), 1-12 (2016).

27. Koberle B, Koch B, Fischer BM, Hartwig A. Single nucleotide polymorphisms in DNA repair genes and putative cancer risk. Arch. Toxicol. 90(10), 2369-2388 (2016).

28. Stracquadanio G, Wang X, Wallace MD et al. The importance of $\mathrm{p} 53$ pathway genetics in inherited and somatic cancer genomes. Nat. Rev. Cancer 16(4), 251-265 (2016).

29. Rybicki BA, Conti DV, Moreira A, Cicek M, Casey G, Witte JS. DNA repair gene XRCC1 and XPD polymorphisms and risk of prostate cancer. Cancer Epidemiol. Biomarkers Prev. 13(1), 23-29 (2004).

30. Tsuchiya N, Matsui S, Narita $S$ et al. Distinct cancer-specific survival in metastatic prostate cancer patients classified by a panel of single nucleotide polymorphisms of cancer-associated genes. Genes Cancer 4(1-2), 54-60 (2013).

31. Lin C-C, Wu H-C, Tsai F-J, Chen H-Y, Chen W-C. Vascular endothelial growth factor gene - $460 \mathrm{C} / \mathrm{T}$ polymorphism is a biomarker for prostate cancer. Urology 62(2), 374-377 (2003).

- Analyzes the importance of HOTAIR single nucleotide polymorphisms in prostate cancer on the Iranian population.

32. Teixeira AL, Ribeiro R, Cardoso D et al. Genetic polymorphism in EGF is associated with prostate cancer aggressiveness and progression-free interval in androgen blockade-treated patients. Clin. Cancer. Res. 14(11), 3367-3371 (2008).

33. Li L, Dang Q, Xie H et al. Infiltrating mast cells enhance prostate cancer invasion via altering LncRNA-HOTAIR/PRC2-androgen receptor (AR)-MMP9 signals and increased stem/progenitor cell population. Oncotarget 6(16), 14179-14190 (2015).

34. Taheri M, Habibi M, Noroozi R et al. HOTAIR genetic variants are associated with prostate cancer and benign prostate hyperplasia in an Iranian population. Gene 613, 20-24 (2017).

35. Bayram S, Ülger Y, Sümbül AT et al. A functional HOTAIR rs920778 polymorphism does not contributes to gastric cancer in a Turkish population: a case-control study. Fam. Cancer 14(4), 561-567 (2015).

36. Bayram S, Sümbül AT, Dadaş E. A functional HOTAIR rs12826786 C $>$ T polymorphism is associated with breast cancer susceptibility and poor clinicopathological characteristics in a Turkish population: a hospital-based case-control study. Tumor Biol. 37(4), 5577-5584 (2015).

37. Yan R, Cao J, Song C et al. Polymorphisms in lncRNA HOTAIR and susceptibility to breast cancer in a Chinese population. Cancer Epidemiol. 39(6), 978-985 (2015).

38. Qi Q, Wang J, Huang B et al. Association of HOTAIR polymorphisms rs4759314 and rs 920778 with cancer susceptibility on the basis of ethnicity and cancer type. Oncotarget 7(25), 38775-38784 (2016). 